\title{
Chapter 9 \\ Conclusion
}

Here we have proposed complex HDA, viz., FRTDSN-HRB for bankruptcy prediction. FRTDSN is the deep learning model based on TDSN which is made up of multiple stacked blocks where the mapping from input to output through weight tensor higher-order statistics. The learning algorithm of TDSN uses weight matrices and tensors for parameter estimation. The computational power of TDSN is increased by incorporating fuzzy rough sets. HRB are formed by probabilistic rough sets in structured hierarchical Bayesian model where hyperparameter and hyperprior are used in order to achieve the posterior distribution. Then FRTDSN is integrated with HRB which results in FRTDSN-HRB model. HRB enhances the prediction accuracy of FRTDSN-HRB model. All the experiments are performed on the Korean construction companies, American and European nonfinancial companies' datasets, and UCI Machine Learning Repository bankruptcy database. FRTDSN-HRB performance is compared with fuzzy SVMs and other statistical models. To provide a balance in comparison, certain aspects that reduce or grow predictive accuracy are taken care through FRTDSN-HRB model. The cutoff points' selection is affected by backcasting, choice-based sample bias, and change of financial ratio. The prediction results are pushed by the sampling procedure. The arbitrariness level in finding cutoff point is reduced. The optimal cutoff point is used which is calculated by using the training sample as proxy for the real optimal cutoff point. This leads to minimum misclassification cost when compared to cutoff points. The empirical optimal cutoff point denotes that all the models are statistically better in comparison superior to the Bayesian model. This is based on the randomly selected samples when the Type I error cost to Type II error cost ratio is not high. The results are highlighted through several important statistical parameters spread across different business cycles, and mid-cycles illustrate the superiority of the proposed FRTDSN-HRB model. The future research of this work involves development of hybrid soft computing based deep learning models by fine-tuning their parameters such that bankruptcy prediction accuracy is further improved. 